\title{
Human muscle afferent responses to tendon taps
}

\section{Effects of variations in fusimotor bias}

K. S.K. M UR T H Y, P. L. G I L D EN B E R G, A N D

W. SEE L I G ER - P E T E R S E N ${ }^{1}$

From the Department of Surgery (Neurosurgery), The University of Texas Medical School at Houston, Houston, Texas, USA

SUMMARY The effect of varying the fusimotor bias on the muscle spindle responses to light tendon taps has been studied in normal human volunteers using surface electrodes at the wrist for recording whole nerve activity. Reinforcement manoeuvres were found to increase the sensitivity of the afferent responses to the mechanical stimulus. Such sensitisation was found to be exhibited more commonly as a decrease in the latency of the peak of the afferent waveform than as an increase in amplitude. Increase in amplitude of the response was seen in cases where the subject was well relaxed and the test muscle quiescent. A change in fusimotor drive was also achieved by asking the subjects to close their eyes voluntarily during the test, thus depriving themselves of the visual feedback. The results under these conditions were found to be variable though showing considerable changes from control recordings. The effect of reinforcemeng manoeuvres may perhaps result in increasing the dynamic fusimotor drive. Such an effect mas be simulated on occluding the blood supply to the test muscle since ischaemia produces aw immediate rise in the rate of afferent discharge. The method of recording is suggested as convenient technique for clinical use.

In a previous paper (Murthy et al., 1978) it was shown that near-synchronous volley of afferent impulses from hand muscles can be elicited using phasic tendon taps, and that such volleys can be recorded with surface electrodes over the ulnar and median nerves at the wrist. It was also concluded that afferent nerve fibres from the muscle spindle primary endings would be the major contributors to the recorded waveform. The effects of changing the fusimotor bias on the afferent response to tendon taps may, therefore, be studied using this technique. Two methods in which fusimotor drive to the muscles may be changed considerably have been used: (1) the Jendrassik manoeuvre (Jendrassik, 1883), and (2) removal of visual feedback. The effects on the tendon reflex of reinforcement manoeuvres are well-documented (Sommer, 1940; Paillard, 1955; Buller and Dorn-

Address for reprint requests: Dr K. S. Krishna Murthy, Division of Neurosurgery, The University of Texas Medical School at Houston, 6400 West Cullen Street, Houston, TX 77030, USA.

'Present address: Neurological Department, General Hospital Eilbek, 2000 Hamburg 76, Federal Republic of Germany.

Accepted 28 September 1977 horst, 1957; Gassel and Diamantopoulos, 1964a, b; Clarke, 1967; Bishop et al., 1968; Gottlieb and Agarwal, 1973). In this paper the effects on the muscle spindle afferent response to light tendon taps will be described.

\section{Methods}

The details of electrode placement and recording procedures have been described by Murthy et al. (1978). The subjects were normal adult volunteers of either sex to whom the details of the experiment were presented before their participation, and their consent obtained.

The reinforcement manoeuvre was performed by the subject as described in the previous paper. Afferent responses were recorded with skin electrodes over the ulnar and median nerves at the wrist and averaged with 32 repetitions of the taps to the tendon of the first dorsal interosseous $N$ $\left(\mathrm{DI}_{1}\right)$ muscle. Control averages were obtained $\Omega$ with the subjects completely relaxed and observing $N$ the stimuli. Test responses were obtained while $\underset{\mathrm{W}}{\mathrm{N}}$ the subject performed a reinforcement manoeuvre 
with the eyes open. A second test required the subjects to close their eyes during the application of the taps, while maintaining a relaxed posture.

\section{EFFECTS OF ISCHAEMIA}

In a few subjects a blood pressure cuff was applied at the elbow to occlude blood supply to the hand muscles. Ischaemia is known to produce an initial excitation of primary muscle spindle endings in animal experiments (Paintal, 1959; Kayaalp et al., 1972; Murthy and Deshpande, 1974). The responses to tendon taps were monitored at various times in a period of three minutes during which the cuff was on. The increase in the sensitivity of the spindle afferent fibres thus produced was compared with the results of tests involving changes in fusimotor bias.

\section{Results}

\section{EFFECT OF REINFORCEMENT MANOEUVRES}

We have reported briefly (Murthy et al., 1978; Fig. 3 ) that the effect of a reinforcement manoeuvre on the response to tendon taps was a sharpening of the afferent waveform. A closer observation of the effects on the afferent waveform (A) showed that while the latency of onset of the afferent waveform remained without any significant change, there was a clear shift of the peak of the afferent response to the left (Fig. 1), indicating a sensitisation of the receptor. Such a facilitation could be only due to an increased fusimotor drive

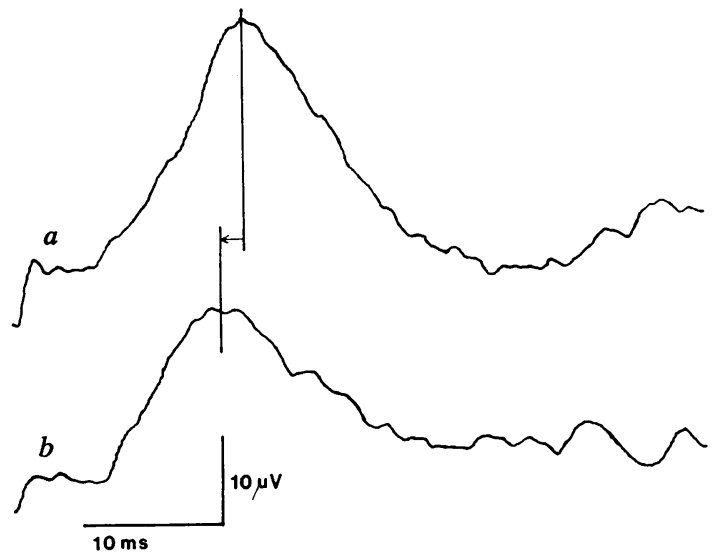

Fig. 1 Effect of reinforcement manoeuvres on the muscle afferent response to tendon taps. Surface electrode recordings at the wrist from ulnar nerve: (a) with subject relaxed, and (b) with subject performing a reinforcement manoeuvre. Note that the peak of the afferent response shifts towards shorter latency during the manoeuvre. Averages of 32 sweeps. since the subjects were instructed to keep the test muscle quiescent (as monitored by a loudspeaker). However, some subjects were not fully relaxed during the experiments as demonstrated by the appearance of a reflex response ( $R$ ) even for light tendon taps (Fig. 2). Even in these subjects a
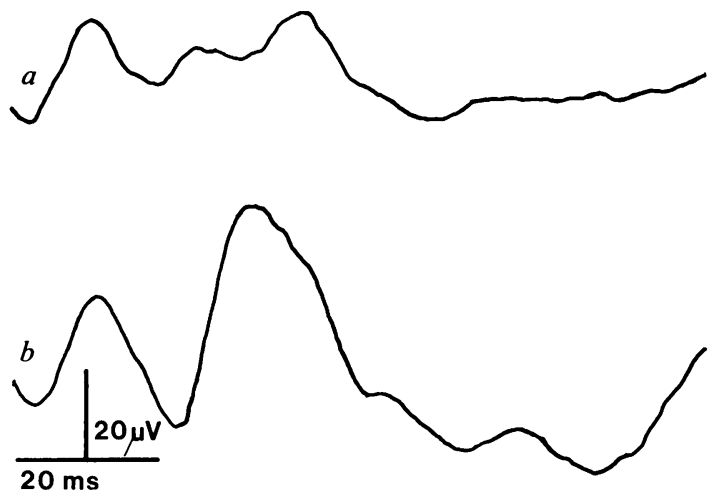

Fig. 2 Similar experiment as in Fig. 1 but with the subject unable to achieve complete relaxation of the test muscle as observed from the appearance of the reflex (a). On performing a reinforcement manoeuvre (b) the reflex response is increased with modification of the afferent waveform. Averages of 32 sweeps.

change in the afferent waveform was easily observed during the reinforcement manoeuvre. There was also a simultaneous increase in the reflex response in such cases. The effect of the reinforcement manoeuvre is to reduce the latency of the peak of the afferent waveform in response to the mechanical stimulus. Such an effect was observed clearly even when the control records showed a biphasic waveform due to some neural activity picked up by the indifferent electrode (Fig. 3).

\section{EFFECT OF ABSENCE OF VISUAL FEEDBACK}

The absence of visual feedback showed different effects in different subjects. Figure 4 illustrates decrease in the latency of the peak of the afferent waveform while Fig. 5 was obtained from another subject exhibiting an increase in the latency of the afferent response. In any event, an increase or decrease in fusimotor drive may be expected as a result of depriving the subject of visual feedback and its effects mediated by the reticular formation. In both cases, however, a decrease in the latency of the reflex response was observed. This is demonstrated in Fig. 5.

EFFECT OF ISCHAEMIA

Figure 6 illustrates the effects of a blood pressure 


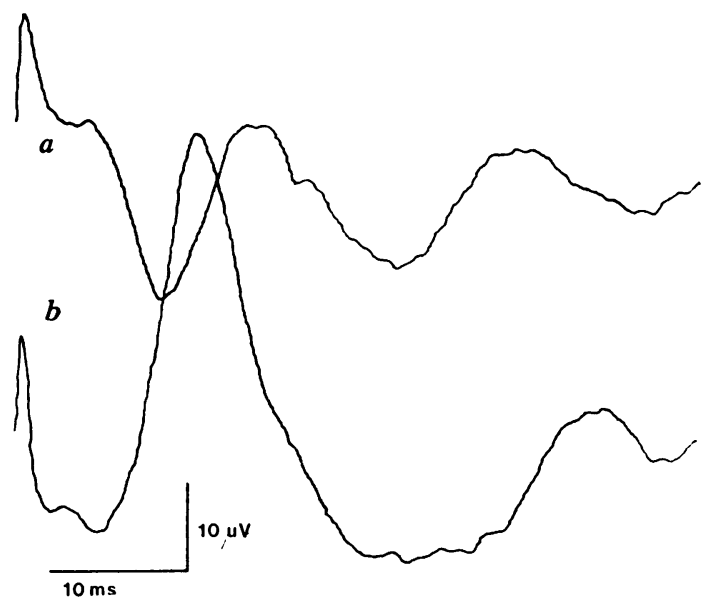

Fig. 3 Experiment with reinforcement manoeuvres in a third subject lying supine on a table. The effect on the afferent response to tendon taps is prominent during the reinforcement manoeuvre (b), although the response obtained while the subject was relaxed (a) displayed a biphasic waveform due to possible contributions from the radial nerve. Averages of 32 sweeps.

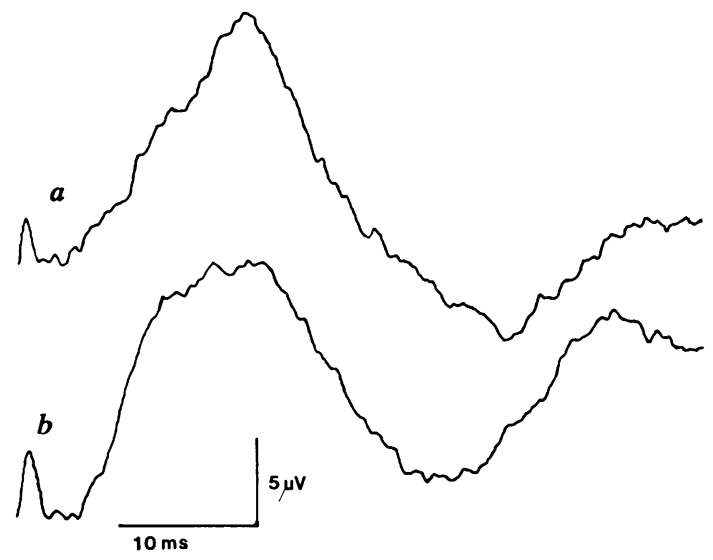

Fig. 4 Effect of loss of visual feedback: (a) control response to taps with eyes open, and $(b)$ response to taps with eyes closed. A verages of 32 sweeps.

cuff applied just above the elbow on the afferent response to tendon taps. Recordings were made simultaneously from the median and ulnar nerves in this subject. The larger response recorded by the median nerve electrode in this subject is probably due to the contributions from the first lumbrical muscle in addition to that from the $\mathrm{DI}_{1}$. It is observed in both $b$ and $d$ that two minutes after the cuff is applied the afferent response is larger, with the peak occurring at a

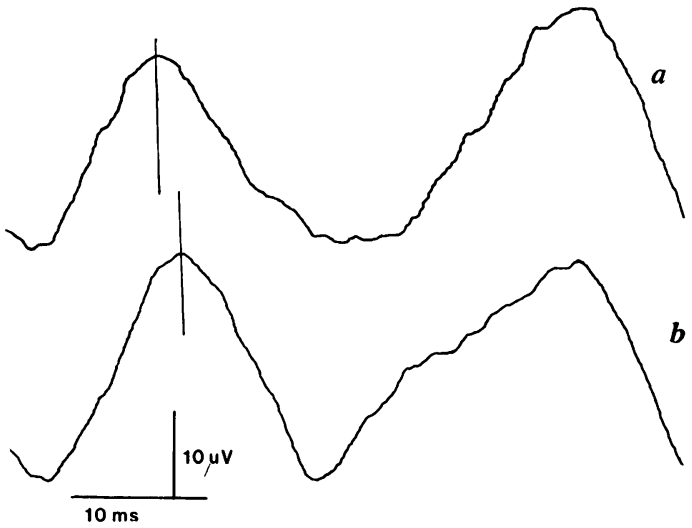

Fig. 5 Effect of loss of visual feedback as in Fig. 4, in another subject to show the variability of response. Averages of 32 sweeps.

shorter latency. Such increases in the afferent response to tendon taps were less obvious if the control response was already large before the $\vec{\omega}$ application of the blood pressure cuff. This was in noticeable particularly when the subject was un $\mathbb{D}$ able to achieve complete relaxation of the hand 음 muscles under test.

\section{Discussion}

The gamma fusimotor innervation of the mam malian muscle spindles gives the central nervous system the capability of controlling the sensitivity of the stretch receptor. It is, therefore, important in problems of neuromuscular control to distinguish situations in which a coactivation of the alpha and gamma motoneurones occurs and those in which the gamma motoneurones are preferentially activated. While this study has not been concerned with the question of the power of the gamma route in eliciting or controlling voluntary movements (Merton, 1953; Matthews, 1972; Stein, 1974), the aim has been to determine fusimotor effects in the human by observing specifically the responses from muscle spindle afferent fibres to a reproducible stimulus. In the previous paper (Murthy et al., 1978) we showed that mechanical tap to the muscle is an adequate stimulus for such a study, and that, by employing surface electrodes and signal averaging techniques, it is possible to record reproducible responses. While the studies employing this technique have not been used for quantitative measurements, a qualitative assessment is still possible. Furthermore, it has been determined that the major contribution to the afferent waveforms recorded in these experiments 

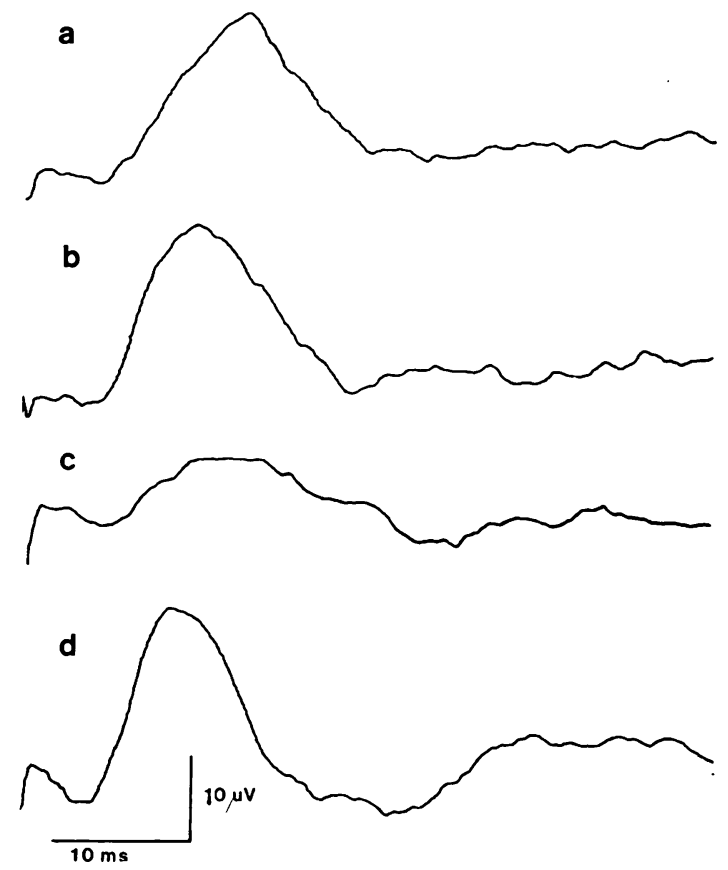

Fig. 6 Effect of ischaemia produced by a blood pressure cuff applied above the elbow. Recording simultaneously from the median nerve $(a, b)$ and the ulnar nerve $(c, d)$ in the same subject. Top traces in both cases are control responses to tendon taps before the cuff was applied. The bottom traces are responses obtained between $1 \frac{1}{2}$ and 2 minutes after the application of the cuff. See text for explanation. Averages of 32 sweeps.

is from the muscle spindle primary endings which are selectively excited by a tendon tap of small amplitude (Lundberg and Winsbury, 1960; Stuart et al., 1970; Matthews, 1972). The secondary endings of the muscle spindle and the Golgi tendon organs are not stimulated by small amplitude tendon taps. The afferent waveform recorded with a surface electrode is equivalent in effect to an integrated multiunit nerve recording. This is due to the capacitive coupling between the electrode and the nerve, caused by the skin and the subcutaneous tissue. The duration of the afferent response is a result of a near synchronous burst of impulses from a number of receptors responding to the phasic mechanical stimulus as has been shown in intranerve recordings (Hagbarth and Vallbo, 1968; Struppler and Erbel, 1972; Hagbarth et al., 1975).

The enhancement of the tendon reflex by reinforcement manoeuvres has been studied in considerable detail, and the contributions have been shown to include gamma fusimotor effects in ad- dition to the direct facilitation of the alpha motoneurones (Gassel and Diamantopoulos, 1964a, b; Clarke, 1967; Bishop et al., 1968; Gottlieb and Agarwal, 1973). However, the fusimotor effects are more dominant (Bishop et al., 1968; Gottlieb and Agarwal, 1973). This was particularly noticeable in this study when the subjects consciously maintained the test muscle quiescent by monitoring its EMG through a loudspeaker. Under these conditions the reflex response to the tendon taps is minimal while the afferent waveform stands out, exhibiting considerable changes during a reinforcement manoeuvre (Fig. 3). It also appears that the fusimotor contribution to the amplitude of tendon reflexes increases in proportion to the strength of the reinforcement manoeuvre itself (Bishop et al., 1968).

In an effort to simulate the increased rate of discharge from spindle primary endings resulting from an increase in fusimotor drive, a blood pressure cuff was applied to the test arm just above the elbow. Ischaemia causes an immediate increase in the rate of impulses from spindle primary endings before suppressing or abolishing their activity (Paintal, 1959; Kayaalp et al., 1972; Murthy and Deshpande, 1974). The results of the experiment illustrated in Fig. 6 may be explained on the basis of such an increase in afferent impulses resulting in an increase in their sensitivity to the tendon taps immediately after the cuff is inflated.

The effect of depriving the subject of visual feedback can interfere with the fusimotor bias through effects mediated by the reticular formation. The effects are probably nonspecific and diffuse, similar to those found in various arousal reactions (Granit, 1975). The results obtained with the subjects studied here (Figs. 4 and 5) have been variable. A detailed study is necessary before the reasons for such variability can be determined.

Using microelectrodes to study the behaviour of human muscle spindle afferent fibres in peripheral nerve fascicles, it has been shown that reinforcement manoeuvres result in activating muscle spindle endings through the fusimotor pathway (Burg et al., 1974; Hagbarth et al., 1975). Szumski et al. (1974) suggested that such effects may be due to selective activation of the dynamic fusimotor neurones. This view has been challenged by Hagbarth et al. (1975) who were unable to find any increase in dynamic sensitivity of the spindle endings during the reinforcement manoeuvres. The results of the studies presented here appear to favour the former view-namely, an increase in the sensitivity of the spindle receptors to the tendon tap. Since only the dynamic fusimotor efferent nerves are effective in increasing the 
spindle sensitivity to a phasic mechanical stimulus, it would be appropriate to conclude that the effects observed during a reinforcement manoeuvre are, at least in part, due to an increase in dynamic fusimotor drive. This increase in spindle sensitivity was not seen as an increase in the afferent response in most cases (Figs. 1 and 2). This is in agreement with the observation made by Hagbarth et al. (1975) from the integrated multiunit activity recorded with microelectrodes. However, the effect is observed in the present studies as a reduction in the latency of the peak of the afferent response, suggesting an increased tendency for synchronous discharge from the afferent fibres.

A contribution from the Golgi tendon organs to the recorded waveform can be ruled out when the test muscle is quiescent since these receptors do not respond to a tendon tap applied to a noncontracting muscle (Lundberg and Winsbury, 1960; Matthews, 1972). In the case of subjects who were unable to achieve complete relaxation of the test muscle, it is possible that some contribution might have resulted from the tendon organs (Fig. 2). This would be an especially significant amount during the reinforcement manoeuvre, since microelectrode recordings from human Ib afferent fibres have shown that their rate of discharge increases during a Jendrassik manoeuvre (Hagbarth et al., 1975). It is not possible to distinguish such a contribution from the results presented in Fig. 2.

The technique presented here can be used in clinical studies on muscle afferent fibres to determine the integrity of their function in various neuromuscular disorders. Since only transcutaneous recording techniques employing standard EMG skin electrodes are used, clinical tests can be easily designed and performed. The results will be complementary to the clinical studies using microelectrode recordings from normal subjects and patients. In the transcutaneous recording technique a multiunit response is obtained. It has been postulated (Murthy and Deshpande, 1974) that, because of their low safety factor for any kind of abnormality affecting the muscle, the primary endings of the muscle spindles are the most likely to be affected earlier during the progress of diseases of the muscle. The present technique will be of use in testing this hypothesis. By evaluating the direct effects on the muscle afferent nerve fibres, it would then be possible to compare such effects with those on $\mathrm{H}$ and $\mathrm{T}$ reflexes.

The authors wish to thank the Fan Kane Research Fund for Brain Injured Children, Tucson, and the Houston Endowment for support and equipment.

\section{References}

Bishop B., Machover, S., Johnstone, R., and Anderson, M. (1968). A quantitative assessment of gamma motoneuron contribution to the Achilles tendon reflex in normal subjects. Archives of Physical Medicine and Rehabilitation, 49, 145-154.

Buller, A. J., and Dornhorst, A. C. (1957). The reinforcement of tendon-reflexes. Lancet, 2, 12601262.

Burg, D., Szumski, A. J., Struppler, A., and Velho, F. (1974). Assessment of fusimotor contribution to reflex reinforcement in humans. Journal of Neurology, Neurosurgery, and Psychiatry, 37, 1012-1021.

Clarke, A. M. (1967). Effect of the Jendrassik manoeuvre on a phasic stretch reflex in normal human subjects during experimental control over supraspinal influences. Journal of Neurology, Neurosurgery, and Psychiatry, 30, 34-42.

Gassel, M. M., and Diamantopoulos, E. (1964a). The Jendrassik maneuver. I. The pattern of reinforcement of monosynaptic reflexes in normal subjects and patients with spasticity or rigidity. Neurology (Minneapolis), 14, 555-560.

Gassel, M. M., and Diamantopoulos, E. (1964b). The Jendrassik maneuver. II. An analysis of the mechanism. Neurology (Minneapolis), 14, 640-642.

Gottlieb, G. L., and Agarwal, G. C. (1973). Modulation of postural reflexes by voluntary movement. Part II. Modulation at an inactive joint. Journal of Neurology, Neurosurgery, and Psychiatry, 36, 540546.

Granit, R. (1975). The functional role of the muscle spindles-fact and hypotheses. Brain, 98, 531-556.

Hagbarth, K-E., and Vallbo, A. B. (1968). Discharge characteristics of human muscle afferents during muscle stretch and contraction. Experimental Neurology, 22, 674-694.

Hagbarth, K-E.. Wallin, G., Burke, D., and Löfstedt, L. (1975). Effects of Jendrassik manoeuvre on muscle spindle activity in man. Journal of Neurology, Neurosurgery, and Psychiatry, 37, 1143-1153.

Jendrassik, E. (1883). Beiträge zur Lehre von den Sehnenreflexen. Deutsche Archiv für Klinische Medizin. 33, 177-199.

Kayaalp, S. O., Kucera, J., and Smith, C. M. (1972). Analysis of muscle spindle afferent responses to norepinephrine and ischemia. In Research in Muscle Development and the Muscle Spindle, pp. 422-436. Edited by B. Q. Banker. R. J. Przybylski, J. P. Van Der Meulen, and M. Victor. Excerpta Medica: Amsterdam.

Lundberg, A., and Winsbury. G. (1960). Selective adequate activation of large afferents from muscle spindles and golgi tendon organs. Acta Physiologica Scandinavica, 49, 155-164.

Matthews, P. B. C. (1972). Mammalian Muscle Receptors and Their Central Actions. Edward Arnold: London.

Merton, P. A. (1953). Speculations on the servocontrol of movement. In The Spinal Cord, pp. 247- 
255. Edited by G. E. W. Wolstenholme. Churchill: London.

Murthy, K. S. K., and Deshpande, S. S. (1974). Differential sensitivity of primary and secondary spindle afferents to depressant drugs. Brain Research.79, 89-99.

Murthy, K. S. K., Gildenberg, P. L., and SeeligerPetersen, W. (1978). Human muscle afferent response to tendon taps. 1 Characteristics of the waveform recorded with transcutaneous electrodes. Journal of Neurology, Neurosurgery, and Psychiatry, 41, 220-225.

Paillard, J. (1955). Réflexes et Regulations d'Origine Proprioceptive chez l'Homme. Arnett: Paris.

Paintal, A. S. (1959). Facilitation and depression of muscle stretch receptors by repetitive antidromic stimulation, adrenaline and asphyxia. Journal of Physiology, 148, 252-266.

Sommer, J. (1940). Periphere Bahnung von Muske- leigenreflexen als Wesen des Jendrassikschen Phänomens. Deutsche Zeitschrift für Nervenheilkunde, 150, 249-262.

Stein, R. B. (1974). Peripheral control of movement. Physiological Reviews, 54, 215-243.

Struppler, A., and Erbel, F. (1972). Analysis of proprioceptive excitability with special reference to the unloading reflex. In Neurophysiology studied in Man. Edited by G. G. Somjen. Excerpta Medica: Amsterdam.

Stuart, D. G., Mosher, C. G., Gerlach, R. L., and Reinking, R. M. (1970). Selective activation of 1a afferents by transient muscle stretch. Experimental Brain Research, 10, 477-487.

Szumski, A. J., Burg, D., Struppler, A., and Velho, F. (1974). Activity of muscle spindles during muscle twitch and clonus in normal and spastic human subjects. Electroencephalography and Clinical Neurophysiology, 37, 589-597. 